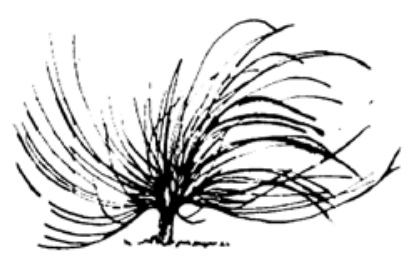

\title{
Intervención educativa en el alumnado con altas capacidades
}

\author{
Lina Higueras-Rodríguez ${ }^{1}$ \\ Universidad de Granada \\ Granada, España \\ mlina@ugr.es
}

\begin{abstract}
Resumen
Este ensayo trata de dar una visión general de aspectos a considerar en la intervención educativa del alumnado con altas capacidades intelectuales. Por ello, trata sobre esta necesidad educativa, las características que presenta el alumnado y cómo se manifiestan. Además de dar un enfoque sobre la atención a la diversidad y la inclusión de este estudiantado dentro de clase y fuera de ella. Un aspecto significativo a resaltar es la importancia que tienen, tanto familias como docentes a la hora de atender las necesidades educativas que presentan estos niños y niñas.
\end{abstract}

\section{(c) (1) (9) $\Theta$}

http://dx.doi.org/10.15359/rep.12-1.4

Recibido: 13 de setiembre de 2016-Aprobado: 9 de mayo de 2017

1 Lina Higueras-Rodríguez es personal de investigación en formación de la Universidad de Granada (España). Miembro del grupo de investigación FORCE y del proyecto "Atlántida, educación y culturas democráticas". Máster en Intervención Psicopedagógica. Las áreas de investigación incluyen: Formación inicial de docentes, herramientas educativas para la enseñanza y el aprendizaje y, actualmente, liderazgo y mejoramiento de la escuela mediante la colaboración en el proyecto Internacional "ISSPP". 
Palabras clave: escuela inclusiva, altas capacidades, rendimiento académico.

\begin{abstract}
This essay tries to give an overview of aspects to consider in the educational intervention of students with high intellectual abilities. Therefore, it addresses this educational need, the characteristics presented by the students and how they manifest themselves besides focusing attention on the diversity and inclusion of these students in class and outside it. A significant aspect to highlight is the importance that both families and teachers have when meeting the educational needs presented by these children.
\end{abstract}

Keywords: inclusive school, high capacities, academic performance

\title{
Introducción
}

$\mathrm{E}$ n la actualidad existe un vacío dentro del sistema educativo que repercute en los niños con altas capacidades intelectuales. Este vacío puede deberse al desconocimiento del personal docente sobre cómo afrontar situaciones dentro del aula en donde se encuentre con este tipo de alumnado que presenta esta necesidad educativa, así como la falta de herramientas y técnicas que necesita el profesorado para el proceso de enseñanza- aprendizaje con estudiantes en esta condición (Calero, García y Gómez, 2007; Fernández, García, García y Fernández, 2001; Torrego y otros, 2011).

Cuando al niño o a la niña con altas capacidades intelectuales le situamos en el ámbito escolar, podemos encontrarnos con profesorado que no dispone de herramientas y estrategias especializadas para tratar con este tipo de alumnado. Esto provoca una disminución en el desarrollo potencial de sus estudiantes y, por lo tanto, se genera fracaso escolar en un alto porcentaje de estos grupos (Gálvez, 2000). Según Pardo de Santoyana (2004), estudiantes con altas capacidades intelectuales, y dificultades de aprendizaje son la población excepcional porcentualmente mayor con respecto a otras. Este alumnado presenta una combinación 
difícilmente apreciable en el aula, lo que puede llevar a una inadecuada adaptación o, incluso, a la inexistencia de respuesta debido a un posible enmascaramiento entre ambas excepcionalidades.

En la sociedad se conocen relativamente muchas de las discapacidades o deficiencias que afectan al campo cognitivo o social, como, por ejemplo, el síndrome de Down, autismo, dislexia, etc. (Inza, Salguero y Fernández, 2016). Sin embargo, las altas capacidades son un campo desconocido para gran cantidad de docentes e incluso llega a ser simplificado hasta el punto en que el niño o la niña que presenta altas capacidades es la persona más lista de la clase y que no requiere que se atiendan sus necesidades (Tojo, Santos, Noles y Quintela, 2017). Según las investigaciones de Seijo, López, Pedrajas y Martínez (2015):

La respuesta satisfactoria a las necesidades únicas de los alumnos con altas capacidades es una preocupación constante para los profesionales de la educación. La intervención educativa nos ofrece alternativas de respuesta, entre ellas el enriquecimiento, encaminado a ofrecer aprendizajes más ricos y variados a través de la modificación en la profundidad del contenido y de la metodología empleada. (p. 27)

Para poder indagar más acerca de esta temática, se va a dar respuesta a una serie de preguntas que ayuden a profundizar y a entender la realidad educativa de estos niños y niñas.

\section{¿Qué entendemos por alumnado con altas capacidades intelectuales?}

Son muchas las clasificaciones que se han hecho a lo largo de la historia con los rasgos de las personas con altas capacidades intelectuales. La idea inicial de la persona superdotada consistía en creer que era inestable emocionalmente, solitaria y débil (Cañete Pulido, 2010). Sin embargo, se demostró que tales fueron solo estereotipos que se han ido desechando a lo largo de diversas investigaciones.

Estos niños y niñas requieren de programas educativos diferenciados y servicios más allá de lo que suministran los programas escolares normales en pos de realizar sus contribuciones a sí y a la sociedad (Cano, 2012). 
Se considera que un alumno o alumna presenta altas capacidades intelectuales "cuando maneja y relaciona múltiples recursos cognitivos de tipo lógico, numérico, espacial, de memoria, verbal y creativo, o bien destaca especialmente y de manera excepcional en el manejo de uno o varios de ellos" (Junta de Andalucía. Plan de actuación para la atención educativa al alumnado con necesidades específicas de apoyo educativo por presentar altas capacidades intelectuales en Andalucía, 2011, p. 8).

La niñez con altas capacidades es un alumnado que presenta necesidades educativas especiales, de ahí la importancia de considerar el desarrollo de programas acordes con dichas necesidades. Autores como Sánchez-Manzano (2009) han señalado que gran cantidad de estudiantes no se identifican a tiempo dentro de los centros educativos y dejan sus estudios por la falta de apoyos, tanto humanos como materiales, encaminados a desarrollar el potencial que presentan.

Dentro del campo educativo, la adaptación curricular que ha sido mejor valorada por docentes es la propuesta que realiza Renzulli que consiste específicamente en:

El enriquecimiento del programa curricular, en el que se propone un aprendizaje innovador, flexible y creativo. Puede realizarse vinculado al programa educativo del alumno y centrarse en una o varias asignaturas y manifestarse en la elaboración de ajustes en cuanto a la profundidad y extensión de los contenidos dentro del aula. Se basa en la noción de que los niños exhiben conductas con talento cuando realizan un proyecto particular o actividades en las que puedan demostrar alta habilidad y creatividad. (Ramírez y Soto, 2016, p. 2)

\section{¿Qué tipo de características presentan? ¿Cómo llegan a manifestarse estas cualidades?}

El alumnado de altas capacidades intelectuales no llega a formar un grupo homogéneo y, por tanto, no se puede llegar a hablar de unas características comunes. Además, la mayoría de este alumnado no muestra todos los rasgos definitorios ni lo hace de forma continuada. Enseguida, se muestran las características más significativas que pueden tener. Para ello, se ha utilizado el Manual de atención al alumnado con necesidades especificas de apoyo educativo por presentar altas 
capacidades intelectuales, de la conserjería de Educación de la Junta de Andalucía (s. f., p.10-11):

\section{Inteligencia:}

- Comprenden y manejan símbolos e ideas abstractas, complejas, nuevas; captan con rapidez las relaciones entre éstas y los principios que subyacen en las mismas.

- Son más rápidos procesando la información. Conectan e interrelacionan conceptos. Poseen y construyen esquemas complejos y organizados de conocimiento, muestran más eficacia en el empleo de procesos meta cognitivos.

- Tienen una capacidad superior para resolver problemas de gran complejidad, aplicando el conocimiento que ya poseen y sus propias habilidades de razonamiento.

- Poseen una gran habilidad para abstraer, conceptualizar, sintetizar, así como para razonar, argumentar y preguntar.

- $\quad$ Presentan gran curiosidad y un deseo constante sobre el por qué [sic] de las cosas, así como una variedad extensa de intereses.

- Tienen una alta memoria.

- Presentan un desarrollo madurativo precoz y elevado en habilidades perceptivo-motrices, atencionales, comunicativas y lingüísticas.

\section{Creatividad}

- Presentan flexibilidad en sus ideas y pensamientos.

- Abordan los problemas y conflictos desde diversos puntos de vista aportando gran fluidez de ideas, originalidad en las soluciones, alta elaboración de sus producciones y flexibilidad a la hora de elegir procedimientos o mostrar opiniones y valorar las ajenas.

- Desarrollan un pensamiento más productivo que reproductivo.

- $\quad$ Poseen gran capacidad de iniciativa.

- Manifiestan creatividad y originalidad en las producciones que realizan (dibujos, juegos, música, etc.).

- Disfrutan de una gran imaginación y fantasía. 


\section{Personalidad}

- Suelen ser muy perfeccionistas y críticos consigo mismos en las tareas y el trabajo que desarrollan.

- $\quad$ Prefieren trabajar solos, son muy independientes.

- Pueden liderar grupos debido a su capacidad de convicción y persuasión y a la seguridad que manifiestan. Con frecuencia muestran gran interés por la organización y manejo de los grupos de trabajo.

- $\quad$ Presentan perseverancia en aquellas actividades y tareas que les motivan e interesan.

- Manifiestan gran sensibilidad hacia el mundo que les rodea e interés con los temas morales y relacionados con la justicia.

- Tienden a responsabilizarse del propio éxito o fracaso. Muestran independencia y confianza en sus posibilidades.

\section{Aptitud académica}

- Realizan aprendizajes tempranos y con poca ayuda. Aprenden con facilidad y rapidez nuevos contenidos y de gran dificultad. Manifiestan interés por adquirir nuevos conocimientos.

- $\quad$ Poseen capacidad para desarrollar gran cantidad de trabajo. Su afán de superación es grande.

- Realizan fácilmente transferencia de lo aprendido a nuevas situaciones y contextos, formulando principios y generalizaciones.

- $\quad$ Tienen gran capacidad para dirigir su propio aprendizaje.

- Comienzan a leer muy pronto y disfrutan haciéndolo.

- Tienen un buen dominio del lenguaje, a nivel expresivo y comprensivo, con un vocabulario muy rico y avanzado para su edad.

- Poseen una mayor facilidad para automatizar las destrezas y procedimientos mecánicos como la lectura, escritura, cálculo...

- Suelen mostrar un elevado interés hacia contenidos de aprendizaje de carácter erudito, técnico o social, dedicando esfuerzos prolongados y mantenidos en asimilarlos y profundizar en ellos y llegando a especializarse en algún tema de su interés. 
5. Aptitud social capacidad de interacción e influencia, apreciable a través de conductas de fácil identificación:

- Capacidad de tomar decisiones.

- Audacia e iniciativa.

- Capacidad de absorber tensiones interpersonales.

- Capacidad para asumir las perspectivas de los otros.

- Elevado punto de mira y razonamiento ético.

- Sensibilidad hacia las necesidades de los demás.

- Disfrute con la relación social (aunque puede preferir la soledad o estar con personas mayores).

- $\quad$ Elevada autoestima (que puede confundirse con presunción).

- Tendencia a influir sobre los demás y a dirigir actividades de grupo.

- $\quad$ Asunción de responsabilidades más allá de lo esperado.

- Aceptación social de su capacidad de influencia.

- Capacidad para resolver problemas de los demás.

- Tenacidad y persistencia en la búsqueda de metas y objetivos.

No es necesario que se den todas estas cualidades simultáneamente para la identificación de un alumno o alumna de alta capacidad, pero sí detectar el mayor número de ellas.

\section{¿Cuáles son las estrategias que se podrían implementar en el con- texto educativo que permitan desarrollar las habilidades de las per- sonas con altas capacidades?}

Cuando hablamos de estrategias que puedan fomentar las habilidades de las personas con altas capacidades, no son solo para este tipo de estudiante, sino que se tiene que tener en cuenta a todo el alumnado en general, con la excepción de que las estrategias y herramientas didácticas que se utilicen puedan adaptarse al tipo de alumnado con el que se trabaje (Higueras-Rodríguez y Fernández, 2017). Las estrategias que se vayan a utilizar para el desarrollo de las actividades, son esenciales para facilitar el logro de los objetivos que se plantean. Es por ello que se debe innovar en el aula, mediante una metodología lúdica, se mejora las habilidades por áreas de desarrollo (Bjorklund y Brown, 1998; Márquez, 2015). Para que se lleve a cabo, hace falta la ayuda de una combinación de metodologías que ayuden a alcanzar los resultados de 
aprendizaje y objetivos previstos (Alonso, Fernández y Nyssen, 2009; Crisol, 2012). Fernández y González (2009) propone una clasificación basada en tres categorías para ubicar las diversas metodologías:

- Métodos de enseñanza basados en las distintas formas de exposiciones magistrales.

- Métodos orientados a la discusión o al trabajo en equipo, juegos cooperativos, grupos interactivos, trabajos en equipo, etc.

- Métodos fundamentados en el aprendizaje individual.

Con respecto a las técnicas y estrategias didácticas es conveniente que el profesorado investigue, seleccione y someta a prueba las más adecuadas de acuerdo con las variables que determinan un ambiente de aprendizaje (Crisol et. al, 2015):

- El estilo docente, cómo ejerce el profesorado la dirección y control del aprendizaje.

- Las tareas de aprendizaje y las tareas de enseñanza.

- Las expectativas que exigen más trabajo y de calidad al estudiantado.

- La cooperación y responsabilidad de estudiantes en las tareas académicas.

- La aceptación positiva entre estudiantes, afecto y no sarcasmos que inhiben el aprendizaje.

- La estructura establecida con respecto a la forma de aprender y a los valores y actitudes que orientan el comportamiento del alumnado.

\section{¿Atender a la diversidad también para quienes destacan por su alta capacidad?}

Para docentes o padres y madres sin la formación suficiente ni la sensibilidad adecuada, el niño o la niña con alta capacidad podría pasar inadvertido, lo que supone que no solo se dejarían de potenciar las cualidades y el desarrollo en todas sus vertientes, sino, lo que puede ser peor, se le estaría negando el derecho de que se atiendan sus necesidades y características particulares e impidiendo el deber que tiene el personal docente y familia al respecto (Hontangas y de la Puente, 2010; Llancavil, 2016). Se considera que sería una gran negligencia por 
la parte docente el no identificar y no tratar atender estas necesidades (Ruíz y Perales, 2017). Se estima conveniente la formación de docentes y familias para que este alumnado no llegue a pasar inadvertido (Martínez-Garrido y Murillo, 2016). Por parte de las familias, es necesaria una formación que abarque esas necesidades fuera del horario escolar a través de propuestas y actividades que ayuden a su hijo o hija en su desarrollo personal, intelectual y social. Por parte de docentes, una formación específica, ya que es este grupo el que adecúa el material, orienta a las familias, etc. Aunque en todo esto no se puede olvidar la labor profesional del equipo de pedagogía ni de orientación educativa. Son un gran apoyo, tanto para docentes como para familias (Ruíz y Perales, 2017).

\section{Conclusiones}

Las personas mejoramos cuando sabemos que los otros individuos esperan eso, o lo que es lo mismo, cuando se tienen altas expectativas sobre nuestro rendimiento. Este, experimenta un notable incremento.

Otro aspecto es el de tratar a cada estudiante como si fuera especial, esto le hará una persona especial, única, orgullosa, con autoestima y amor propio y, además, con identidad; así, fortalecerá su personalidad y será mejor persona.

Otro asunto a considerar es el grado de implicación de las familias, puesto que estas son el primer agente socializador de la niñez. Si estas familias muestran interés en la educación de sus hijos e hijas, pueden llegar a aportar ideas útiles en su aprendizaje. Sin embargo, a estas familias les puede surgir ciertas dudas acerca de la educación de su gente. Algunas de esas dudas son la preocupación por el fracaso escolar porque algunos de estos niños o niñas se aburren en clase, se distancian de sus pares, etc. Además, necesitan una aportación de ideas para que sean capaces de solventar las necesidades fuera del horario escolar, puesto que, cuando llegan a casa, estos niños y niñas quieren indagar, investigar, etc. y estos padres y madres quieren saber qué deben hacer frente a estas situaciones. Por ello, se podría crear una escuela de padres y madres para que las familias puedan ir y formarse para llegar a ser más eficaces.

Tras la búsqueda de información e indagación sobre las posibles estrategias que utiliza la familia para la mejora del aprendizaje de sus hijos, se podría dar una propuesta educativa que abarcara a todos los 
padres y madres con la finalidad de mejorar el aprendizaje de sus hijos. Esta propuesta podría ser la creación, a nivel local, de grupos de familias (Fresnillo y otros, 2000), donde podrían ir a formarse y a compartir sus experiencias con otras personas para que su relación fuera más enriquecedora, es decir, un espacio de información, formación y reflexión dirigido a padres y madres. Dentro de estos grupos, se ofrecerían cursos de formación sobre cómo mejorar la autoestima, sobre educación para la salud, educación en el tiempo de ocio, cómo favorecer la creación de los hábitos de estudio, relaciones sociales y afectivas, control emocional para padres y madres, educación en valores para la convivencia y ciudadanía entre otros cursos.

Para dar respuesta desde el ámbito escolar, sería posible, a través de un currículum flexible y abierto. A través de las adaptaciones curriculares se puede ampliar y enriquecer. Todo esto implica un proceso de doble vía, de ida y vuelta constantemente en función de las necesidades educativas que presente el alumnado. Otero (2009) propone una serie de recomendaciones que ayudan a las adaptaciones curriculares. Estas son: En cuanto a los objetivos, se deben adecuar para la mejora de las habilidades sociales y de las estrategias cognitivas de aprendizaje y metacognición de este estudiantado; en los contenidos se llevará a cabo una ampliación vertical y horizontal, así como la introducción de contenidos diferentes; en lo referente a las actividades, estas deben tener diferentes grados de dificultad y realización, deben existir diferentes actividades para trabajar el mismo contenido y que permitan diversas posibilidades de ejecución. La evaluación será de dos tipos, inicial y por criterios.

Además de estas estrategias instruccionales, también debemos atender las diferentes estrategias organizativas dentro del aula. El agrupamiento de estudiantes es una importante estrategia organizativa que ayuda a que quienes presentan altas capacidades puedan trabajar con otros compañeros y compañeras.

Para finalizar, decir que, al alumnado, independientemente de las características y necesidades educativas que presente, se le debe ayudar a encontrar y utilizar los recursos que tenemos a mano, abrir nuevas puertas y derribar obstáculos en su aprendizaje. El personal docente es un guía que orienta al estudiantado, no necesariamente su fuente de conocimiento. 


\section{Referencias}

Alonso, L. E., Fernández, C. J., \& Nyssen, J. M. (2009). El debate sobre las competencias. Una investigación cualitativa en torno a la educación superior y el mercado de trabajo en España. Madrid: ANECA.

Bjorklund, D. F., \& Brown, R. D. (1998). Psysical play and cognitive development: Integrating activity, cognition and education. Child Development, 69(3), 604-606.

Calero García, M. D., García Martín, M. B. y Gómez Gómez, M. T. (2007). El alumnado con sobredotación intelectual. Conceptualización, evaluación y respuesta educativa. Sevilla: CECJA. Recuperado de http://www.juntadeandalucia.es/averroes/averroes/ $\mathrm{html} /$ portal/com/bin/contenidos/B/ProfesoradoEnRed/NecesidadesEducativasEspeciales/Seccion/AltasCapacidades/ContenidoAltasCapacidades/1206451544236_wysiwyg_libroelalumnadoconsobredotacion.pdf

Cano, I. (2002). Alumnado con altas capacidades intelectuales: Propuesta de intervención (Trabajo final de maestría). Universidad internacional de la Rioja. Recuperado de https://reunir.unir.net/ bitstream/handle/123456789/1032/2012_11_07_TFM_ESTUDIO_DEL_TRABAJO.pdf? sequence $=1$

Cañete Pulido, M. (2010). La educación en niños y niñas superdotados. Revista digital de innovación y experiencias educativas, 30. Recuperado de http://www.csi-csif.es/andalucia/modules/ mod_ense/revista/pdf/Numero_30/MARIA_DEL_MAR_CANETE_PULIDO_01.pdf

Crisol, E. (2012). Opinión y percepción del profesorado y de los estudiantes sobre el uso de las metodologías activas en la Universidad de Granada (Tesis doctoral). Universidad de Granada. Recuperado de http://0-hera. ugr. es. adrastea. ugr. es/tesisu$\mathrm{gr} / 21224043$. pdf).

Crisol, E., Higueras-Rodríguez, L., Amber, D., Barrero, B.,Hinojosa, E., Morales, A., Olmo, M., Pedrosa, B., Quirante, R., Romero, M. A. (2015). Estrategias y métodos de enseñanza- aprendizaje. En J. Domingo y M. Pérez Ferra (Coords.), Aprendiendo a enseñar. Manual práctico de didáctica. Madrid: Pirámide. 
Fernández Abascal, M. T., García Gálvez, C., García Latorre, J. y Fernández Mota, M. E. (2001). Guía para la atención educativa a los alumnos y alumnas con sobredotación intelectual. Sevilla: CECJA. Recuperado de http://www.juntadeandalucia.es/averroes/ averroes $/ \mathrm{html} /$ portal $/ \mathrm{com} / \mathrm{bin} /$ contenidos/B/ProfesoradoEnRed/ NecesidadesEducativasEspeciales/Seccion/AltasCapacidades/ ContenidoAltasCapacidades/1206451504676_wysiwyg_guiaalumandosobredotacionintelectual.pdf

Fernández, M. D., y González, A. S. (2009). Estrategias didácticas creativas en entornos virtuales para el aprendizaje. Revista Electrónica publicada por el Instituto de Investigación en Educación.

Fresnillo, V., Fresnillo, R., y Fresnillo, M. (2000). Escuela de padres. Recuperado de http://orientacionandujar.files.wordpress. com/2009/05/escuela-de-padres.pdf

Gálvez, J. M. (2000). Alumnos precoces, superdotados y de altas capacidades. Ministerio de Educación.

Higueras-Rodríguez, L., y Fernández Gálvez, J. D. (2017). El papel de la familia en la educación de los niños con altas capacidades intelectuales. International Journal of Educational Research and Innovation, 7, 149- 163.

Hontangas, N. A., y de la Puente, J. L. B. (2010). Atención a la diversidad y desarrollo de procesos educativos inclusivos. Prisma Social: Revista de Ciencias Sociales, (4), 13.

Inza, M. Á. G., Salguero, F. L., y Fernández, V. L. (2017). Mejora del rendimiento académico de alumnos con necesidades específicas de apoyo educativo mediante una metodología innovadora desarrollando la creatividad y su relación con la lateralidad en educación primaria. Revista de Educación Inclusiva, 9(1).

Junta de Andalucía. Consejería de Educación. (2011). Plan de actuación para la atención educativa al alumnado con necesidades especificas de apoyo educativo por presentar altas capacidades intelectuales en Andalucia. Recuperado de http://www.juntadeandalucia.es/export/drupaljda/Planaltas.pdf

Junta de Andalucía. Consejería de Educación. (s. f.). Manual de atención al alumnado con necesidades específicas de apoyo educativo por presentar altas capacidades intelectuales. Conserjería de Educación de la Junta de Andalucía. Recuperado de http:// 
www.juntadeandalucia.es/educacion/webportal/ishare-servlet/ content/5f4b7f67-d053-4ee8-9de9-4a7295134e96

Llancavil, D. L. (2016). The importance of Inclusive Education for work with children with academic talent. Perspectiva Educacional, 55(1), 168-183.

Márquez, I. (2015). El desarrollo de las capacidades comunicativas y sociales en niños de 2-3 años durante el juego (Tesis de grado) Pontificia Universidad Católica del Ecuador, Ciencias de la Educación, Ecuador. Recuperado de http://repositorio.puce.edu.ec/ handle $/ 22000 / 8464$

Martínez-Garrido, C., y Murillo, F. J. (2016). Investigación iberoamericana sobre enseñanza eficaz. Revista mexicana de investigación educativa, 21(69), 471-499.

Otero Martínez, N. (2009) ¿Qué hacer con un alumno con altas capacidades?. Cuadernos de Educación y Desarrollo, 1(9). Recuperado de http://www.eumed.net/rev/ced/09/nom5.htm

Pardo de Santoyana, R. (2004). Alumnado doblemente excepcionales: Superdotación intelectual y dificultades de aprendizaje. FAISCA: Revista de altas capacidades, 11, 37-46.

Ramírez, F. Z., y Soto, B. I. C. (2016). Evaluación de la eficacia de tres programas de enriquecimiento de la creatividad. RIDPSICLO, 2(2), 6.

Ruiz, A. P., y Perales, R. G. (2017). Innovación y creatividad para favorecer la intervención educativa del alumnado con altas capacidades. Revista de Educación Inclusiva, 9(1).

Sánchez- Manzano, E. (2009). La superdotación intelectual. Málaga: Aljibe.

Seijo, J. C. T., López, C. M., Pedrajas, M. L., y Martínez, C. (2015). Formación del profesorado en aprendizaje cooperativo y alumnos con altas capacidades: Un enfoque inclusivo. Revista Latinoamericana de educación inclusiva, 9(2), 91-110.

Tojo, C. M. P., Santos, G. C., Nores, A. I., Cao, L. R., y Quintela, L. R. (2017). Los programas de enriquecimiento: Desarrollo integral de las altas capacidades. International Journal of Developmental and Educational Psychology. Revista INFAD de Psicología., 6(1), 27-32. Torrego, J. (Coord.). (2011). Alumnado con altas capacidades y aprendizaje cooperativo. Madrid: Fundación SM. 